\title{
"Numbers sometimes lie"- refractive surprise following IOL mislabeling by the manufacturer
}

\author{
Marija Antičić $^{1} \cdot$ Navid Ardjomand ${ }^{2} \cdot$ Stephanie Sarny $^{1} \cdot$ Jakob Schweighofer $^{1} \cdot$ Yosuf El-Shabrawi $^{1}$
}

Received: 18 January 2019 / Accepted: 25 January 2019 / Published online: 13 February 2019

(C) The Royal College of Ophthalmologists 2019

Accurate biometry, appropriate intraocular lens (IOL) power formula, and modern techniques are very important for cataract surgery as a refractive procedure aiming at the intended refractive result.

Main sources of postoperative refractive surprise are errors in biometry, such as, inaccurate preoperative measurements (axial length [AL] or keratometry), IOL calculation formula, IOL insertion, and lens constant's errors [1]. Other common reasons for 'wrong' IOL implantation as reported by Steeples in their study of 178 cases are: inaccurate biometry, wrong IOL selection, transcription errors, handwriting misinterpretations, change in list order, right/left eye confusion, patient identification issues, misfiled biometry, wrong IOL written on theater white board, optimal IOL power unavailable in stock, wrong IOL power implantation after complicated surgery, wrong patient notes, communication errors, or - as in the case presented herein an IOL mislabeled by the manufacturer [2].

In case of a refractive surprise following cataract surgery, identification of the failure cause will lead us to an optimal solution.

Corneal irregularities, long-term history of contact lens use, superficial punctate keratitis, posterior capsular opacity, decentered IOL, preoperative inaccurate measurement, implantation of the wrong IOL, intraoperative possible upside-down implantation, or fundus problems have to be excluded [3].

Corneal topography and corneal tomography often provide the best overall assessment of the corneal status and degree of astigmatism, irregularity, and symmetry, and

Marija Antičić

marijanticic@gmail.com

1 Department of Ophthalmology, General Hospital Klagenfurt, Klagenfurt, Austria

2 Department of Ophthalmology, Medical University of Graz, Graz, Austria should exclude corneal pathologies like mild keratoconus [4]. Another reason for implementing Scheimpflug imaging is the fact that central IOL thickness measurements correlate closely with IOL power [5]. Thus the actual IOL power can be determined in situ, in an already implanted IOL.

When dealing with mild ametropia after cataract surgery, a manufacturer's error is rarely considered as a cause for the refractive surprise.

A 65-year-old male patient with nuclear sclerosis grade II in both eyes, and best corrected visual acuity of 6 out of 9 in the right eye and 6 out of 12 in the left eye, was referred for the cataract surgery in the left eye. Preoperative biomicroscopy and dilated fundus examination were unremarkable. Biometry for IOL power calculation was done using the IOL Master 500 system (Carl Zeiss Meditec AG, Jena, Germany). The IOL power calculated for the left eye was +19.5 diopter (D) (target residual refractive error $-1.16 \mathrm{D}$ ). The patient underwent uneventful phacoemulsification with implantation of monofocal hydrophobic aspheric IOL (Zeiss LUCIA 611P/PY) of $+19.5 \mathrm{D}$ under peribulbar anesthesia. His postoperative visual acuity 1 day after surgery was counting fingers at $1 \mathrm{~m}$ improving to 6 out of 9 with refraction of $-14.0 \mathrm{D}$. A detailed biomicroscopy revealed normal anterior segment with well-centered in-the-bag IOL. Capsular distension syndrome and tilted or subluxated IOL were ruled out. A dilated fundus examination ruled out posterior segment pathology. Repeated biometry confirmed the IOL power to be $+19.5 \mathrm{D}$ which was the same as the power of the lens implanted at the time of the surgery.

Patient was examined with the Pentacam HR Scheimpflug camera (Oculus Optikgeräte GmbH, Wetzlar, Germany) to rule out undetected keratoconus. Examination confirmed regular and symmetric corneal tomography. After excluding all other possible pitfalls, we started to speculate on mislabeling of the IOL by the manufacturer as the potential reason of the enormous postoperative refractive error.

As we suspected wrong labeling of the IOL by the manufacturer, we focused once more to the Pentacam analysing IOL thickness with Scheimpflug imaging to calculate 


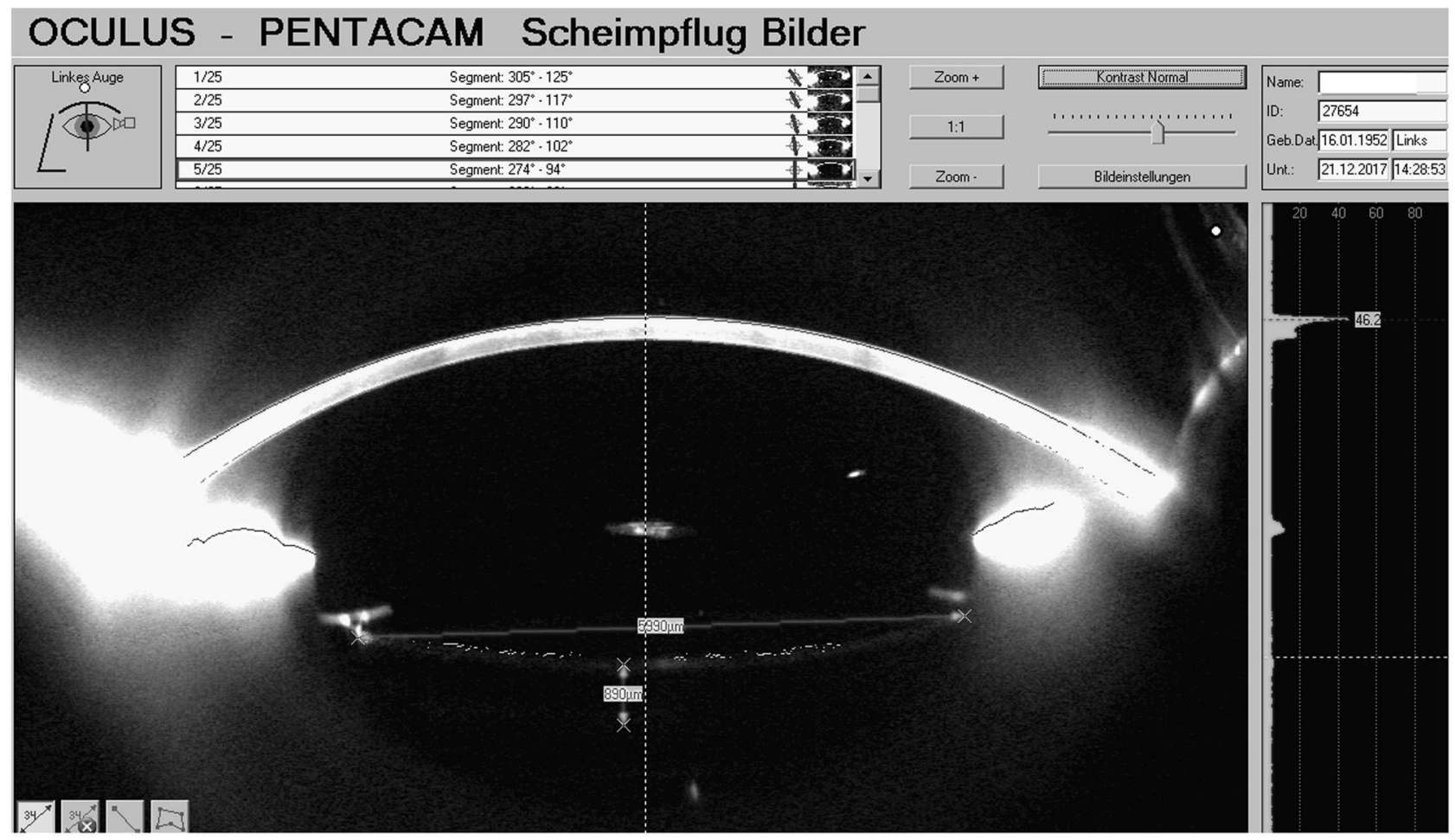

Fig. 1 Scheimpflug image of lens thickness in correlation with lens power on Pentacam (Oculus Optikgeräte GmbH, Wetzlar, Germany) prior to the IOL exchange

IOL power. Central IOL thickness measurement with the Pentacam Scheimpflug camera closely correlates with the IOL power [5]. Using Scheimpflug imaging, the lens thickness of the implanted IOL was determined to be $890 \mu \mathrm{m}$ (Fig. 1). When we implemented this thickness into the formula: lens power $=(0.045 \times 890)-6.549$ (ref. 5), our measurement indicated that a $+34.0 \mathrm{D}$ IOL was implanted instead of +19.0 D. An IOL exchange was advised to the patient.

Written informed consent for publication was obtained from the patient after surgery and approval from the Institutional Review Board (General Hospital Klagenfurt, Klagenfurt, Austria) was granted.

Biometry was repeated for IOL of different manufacturer. Intraoperatively, the IOL was cut with IOLExplantation-Set scissors (Snyder/Osher IOL scissors, GEUDER AG, Heidelberg, Germany) and explanted through a 2.4-mm temporal clear corneal incision after the endothelium was coated and the bag filled with a dispersive ophthalmic viscosurgical device. A $+18.0 \mathrm{D}$ aspheric monofocal IOL (Vivinex XC1/XY1, Hoya Surgical Optics, Singapore) was implanted in the bag, aiming for target residual refractive error of $-0.82 \mathrm{D}$.

The explanted IOL was sent to the manufacturer, where it was confirmed that a +34.0 D IOL was packed, instead of a +19.5 D IOL. One day postoperatively, the CDVA was 20 out of 20 with a manifest refraction of $-0.75 \mathrm{D}$.
There is a paucity of reports on mislabeled IOL as a cause of refractive surprise. Only two case reports have been published in 2000 and 2012 [6, 7]. Central IOL thickness measured with the Pentacam Scheimpflug camera correlates significantly with the known IOL power, as reported by Turner et al. [5]. This article was key which gave us direction how to measure present IOL power before its explantation.

By employing Scheimpflug imaging, we were able to get an estimate of the actual IOL implanted, thereby indicating a mislabeled IOL. Following this occurrence, the manufacturer did change their safety procedures to ensure that this remains a one-time case.

Significant amounts of residual error after cataract surgery is more common when an eye is highly ametropic, because biometry gets less accurate. After we excluded possible causes such as an undiagnosed keratoconus prior the cataract surgery, decentered IOL, posterior capsular opacity, inaccurate biometry, upside-down IOL implantation and fundus problems on IOL exchange was performed.

When we deal with mild ametropia after cataract surgery, we usually never consider a manufacturer's error as the cause of a refractive surprise. We usually recheck all our surgical steps. In our case, however, due to the enormous postoperative refractive error, after excluding all possible causes, we had suspected mislabeling of the IOL by 
manufacturer. In our Department we always use preoperative cataract-specific checklists with local personalization and adaptation. Additionally, our cataract surgeons undertake a pre-operative team brief at the start of a cataract surgery, which provides a vital final opportunity to check the patient, site, procedure, and the IOL required against source biometry documents before each operation.

Wrong IOL implants are the leading cause of wrong implant never events [8]. Surgical checklists have been widely adopted, and

are mandatory in many healthcare systems, following demonstration of improvement in surgical morbidity and mortality [2].

Steeples et al. demonstrate the persistence of wrong IOL events in the NHS in England and Wales, despite promotion of patient safety and mandatory use of surgical checklists [2]. In his work only one case of wrong IOL was caused by wrong IOL in the wrong box. However, reputational damage of the company and financial penalties associated with mislabeled IOL by the manufacturer may lead to under-reporting.

Experience demonstrates that checklists alone are not completely effective in preventing adverse incidents [2]. Furthermore, analysis of the causal factors suggests that non-technical errors (human factors) are the predominant cause of mistakes [9].

The IOL and its label are checked many times. However, the IOL goes through many hands, and humans are responsible for the final labeling [6].

There are different methods that can be used to resolve refractive surprise after cataract surgery, such as cornealbased surgery (laser refractive surgery) and lens-based procedures (IOL exchange or piggyback IOLs) [10].

If the residual error is high and not treatable with corneal refractive surgery, IOL exchange or piggyback IOLs are required. A retreatment is often associated with the risk of multiple complications like: corneal endothelial decompensation, posterior capsular rent, vitreous loss, and unsatisfactory visual outcome [7]. From an aspect of the patient it is even more unpleasant if he has only one functional eye and for the treating surgeon this could lead to medicolegal issues.
In conclusion, when we deal with refractive surprise after cataract surgery, we should add mislabeling of IOL as a potential factor precluding emmetropia. Scheimpflug imaging could be applied clinically in cases of unexplained postoperative refractive error.

\section{Compliance with ethical standards}

Conflict of interest The authors declare that they have no conflict of interest.

Publisher's note: Springer Nature remains neutral with regard to jurisdictional claims in published maps and institutional affiliations.

\section{References}

1. Karabela Y, Eliacik M, Kocabora MS, Erdur SK, Baybora H. Predicting the refractive outcome and accuracy of IOL power calculation after phacoemulsification using the SRK/T formula with ultrasound biometry in medium axial lengths. Clin Ophtalmol. 2017;11:1143-9.

2. Steeples LR, Hingorani M, Flanagan D, Kelly SP. Wrong intraocular lens events-what lessons have we learned? A review of incidents reported to the National Reporting and Learning System: 2010-4 versus 2003-10. Eye. 2016;30:1049-55.

3. Chan TC, Lok JK, Jhanji V, Wong VW. Intraocular lens explantation in Chinese patients: different patterns and different responses. Int Ophtalmol. 2015;35:679-84.

4. Safarzadeh M, Nasiri N. Anterior segment characteristics in normal and keratoconus eyes evaluated with a combined Scheimpflug/Placido corneal imaging device. J Curr Ophtalmol. 2016;28:106-11.

5. Turner SJ, Lee EJK, Hu V, Hollic EJ. Scheimpflug imaging to determine intraocular lens power in vivo. J Cataract Refract Surg. 2007;33:1041-4.

6. Stephan Kohnen MD. Postoperative refractive error resulting from incorrectly labeled intraocular lens power. J Cataract Refract Surg. 2000;26:777-8.

7. Ravi K, Senthil S, Pesala V. Refractive surprise following implantation of correct powered intraocular lens-a real surprise! Int Ophtalmol. 2012;32:603-5.

8. Zamir E, Beresova-Creese K, Miln L. Intraocular lens confusions: a preventable "never event" The Royal Victorian Eye and Ear Hospital protocol. Surv Ophtalmol. 2012;57:430-47.

9. Azuara-Blanco A, Reddy A, Wilkinson G, Flin R. Safe eye surgery: non-technical aspects. Eye. 2011;25:1109-11.

10. Abdelghany AA, Alio JL. Surgical options for correction of refractive error following cataract surgery. Eye Vis. 2014;1:2. 